\title{
The role and gene expression profile of SOCS3 in colorectal carcinoma
}

\author{
Xing Dong ${ }^{1,2}$, Jing Wang ${ }^{3}$, Bo Tang ${ }^{1}$, Yeng-Xue Hao ${ }^{1}$, Ping-Yang Li ${ }^{1}$, Shi-Yong Li ${ }^{2}$ \\ and Pei-Wu Yu ${ }^{1}$ \\ ${ }^{1}$ Department of General Surgery, Southwest Hospital, Third Military Medical University, Chongqing, China \\ ${ }^{2}$ Department of General Surgery, PLA Army General Hospital, Beijing, China \\ ${ }^{3}$ Department of Obstetrics and Gynecology, PLA Army General Hospital, Beijing, China \\ Correspondence to: Pei-WU Yu, email: yupeiwu01@sina.com \\ Keywords: SOCS3; colorectal carcinoma; mechanisms; indicator; gene therapy \\ Received: September 12, 2017 Accepted: December 04, 2017 Epub: December 20, 2017 Published: March 23, 2018
}

Copyright: Dong et al. This is an open-access article distributed under the terms of the Creative Commons Attribution License 3.0 (CC BY

3.0), which permits unrestricted use, distribution, and reproduction in any medium, provided the original author and source are credited.

\section{ABSTRACT}

SOCS3 has been postulated to play a role in the occurrence and progression of malignancies. However, the relationship of SOCS3 with colorectal carcinoma remains poorly understood. The purpose of the study was to explore the role of SOCS3 in colorectal carcinoma and its underlying mechanisms. Protein and mRNA expression of SOCS3 in colorectal carcinoma and normal colorectal mucosa was detected using immunohistochemistry and real-time quantitative PCR. SOCS3 expression was significantly lower in colorectal carcinoma tissue than in normal colorectal mucosa, and was negatively correlated with tumor invasion depth, lymph node metastasis, differentiation degree, and TNM stage. A stably transfected colorectal carcinoma cell line (8348SOCS3) with high expression of SOCS3 was established. The effects of SOCS3 overexpression on the growth, proliferation, invasion and tumor formation of colorectal carcinoma cells were examined by CCK-8 assay, transwell method and tumorigenicity assays in nude mice. Then we found SOCS3 overexpression significantly decreased proliferation and invasion capability of 8348 cells in vitro and in vivo. Furthermore, the effect of SOCS3 overexpression on the gene expression profile of colorectal carcinoma cells was analyzed using human genome arrays. The results revealed 369 genes that were differentially expressed in 834850 CS3 cells. 193 genes was significantly increased and 176 genes was significantly decreased. Bioinformatics analysis demonstrated that high SOCS3 expression affected multiple signaling pathways in colorectal carcinoma including TGF- $\beta /$ Smads, NF-kB, and HIF-MAPK pathways. Especially for the TGF- $\beta /$ Smads pathways, high SOCS3 expression could inhibit TGF- $\beta 1$ expression and activate Smad4 expression. These data suggested that low expression of SOCS3 was associated with the occurrence and progression of colorectal carcinoma. SOCS3 protein may be a useful indicator for malignancy and prognosis of colorectal carcinoma and also a new target for gene therapy.

\section{INTRODUCTION}

Colorectal carcinoma (CRC) is one of the most common clinical gastrointestinal tumors and its prevalence is increasing annually $[1,2]$. The pathogenesis of CRC and its metastases remains unclear, and there is currently no effective treatment for this malignancy.
Studies have confirmed that CRC is a multi-stage disorder caused by multiple factors and multiple genetic variations and involving the activation of oncogenes, inactivation of tumor suppressor genes, and disorders of apoptosis $[3,4]$. The suppressor of cytokine signaling (SOCS) family plays a key role in regulating the growth and differentiation of cells. In particular, the role of the 
SOCS family in the pathogenesis of malignant tumors has increasingly been recognized [5-7]. As a key member of the SOCS family, SOCS3 participates in the regulation of multiple signaling pathways. In our previous study, we showed that gene silencing of SOCS3 by siRNA significantly improved the erythroid development of human hematopoietic stem cells [8]. Numerous studies have shown that SOCS3 expression can be induced by a variety of pro- and anti-inflammatory cytokines. It can suppresses the signaling of multiple immune molecules. Since it exerts key roles in the development of inflammatory diseases, viral infections, obesity and cancer, SOCS3 may be a biomolecular indicator for disease diagnosis and prognostic prediction. It is also serve as a potential target for the treatment of a variety of diseases $[9,10]$. Research has shown that expression of SOCS-3 is decreased in many malignant tumor tissues and cell lines, such as head and neck squamous cell carcinoma, liver cancer, renal carcinoma, melanoma, and prostate cancer [11-13]. whereas demethylation or recovery of SOCS-3 expression via approaches such as drug treatment can significantly inhibit the growth of tumor cells, suggesting that SOCS-3 may act as a tumor suppressor gene.

To date, few studies have explored the role of SOCS3 in the invasion and metastasis of $\mathrm{CRC}$ and the underlying mechanisms. In the current study, we investigated the roles of SOCS3 protein in the pathogenesis of CRC at cellular and organismal levels and elucidated relevant regulatory signals and pathways. Our study revealed action mechanisms of SOCS3 and provided a new predictive indicator and potential therapeutic target for CRC.

\section{RESULTS}

\section{Expression of SOCS3 in CRC and its significance}

Expression of SOCS3 in CRC and normal colorectal tissue by immunohistochemical method

Positive SOCS3 expression was localized in the cytoplasm (Figure 1A-1B). The expression of SOCS3 in CRC tissue $(32.5 \%, 13 / 40)$ was significantly lower than that in normal colorectal tissues $(77.5 \%, 31 / 40)\left(\chi^{2}=\right.$ 16.364, $P<0.01)$. SOCS3 expression in CRC tissue was not correlated with gender, age, and tumor size (all $P>$ 0.05 ), but was negatively correlated with invasion depth, lymph node metastasis, differentiation degree, and TNM stage (all $P<0.05)$ (Table 1$)$.

\section{Quantitative expression of SOCS3 in CRC and normal colorectal tissues by qRT-PCR}

The expression of SOCS3 mRNA in CRC tissues $(0.467 \pm 0.113)$ was significantly lower than that in normal colorectal mucosa $(1.035 \pm 0.312)(\mathrm{t}=10.826, P<0.01)$ (Figure 1C).

Effect of SOCS3 overexpression on the biological features of CRC cells

\section{Generation of CRC cell line with stable high SOCS3 expression}

\section{Packaging of lentivirus}

During packaging of the 293 cells to produce lentivirus, the envelope glycoprotein could fuse the 293 cells; as a result, the formation of multinucleated syncytia could be seen under light microscopy (Figure 2A). Expression of GFP in 293 cells could be observed under a fluorescence microscope. As shown in Figures 2A and 2B, expression of GFP was observed in more than $95 \%$ of the 293 cells after $72 \mathrm{~h}$.

\section{Transfection of lentivirus into 8348 cells and screening of positive cells}

The 8348 cells were plated in 6-well plates. After cell density reached $70 \%$, we added culture medium containing viral particles and an appropriate amount of polybrene and the cells were cultured at $37^{\circ} \mathrm{C}$ for $12-16$ hours. After the viral supernatant was discarded, the cells were cultured in 8348 growth medium for another $48 \mathrm{~h}$. Fluorescence microscopy showed that the percentage of 8348 cells with expression of GFP was less than $30 \%$. To obtain cells that could stably express SOCS3 expression vector, flow cytometry was performed to sort the transfected 8348 cells based on the expression profile of GFP. As shown in Figure 2C-2E, after sorting the percentage of cells expressing GFP was greater than $90 \%$.

\section{Comparison of SOCS3 expression in 8348SOCS3 cells and 8348plv cells by western blotting and qRT-PCR}

The expression of SOCS3 mRNA and protein was significantly higher in 8348 SOCS3 cells than that in 8348plv cells (Figures 3A-3B), confirming generation of a CRC cell line with stable high SOCS3 expression.

\section{Effect of high SOCS3 expression on the growth and proliferation of CRC cells by CCK-8 method}

8348SOCS3 cells showed a significantly slower proliferation rate than control cells. After transfection for 1,5 , and 7 days, there was a significant difference in the proliferation rate between the 8348 SOCS3 group and 8348plv group $(\mathrm{t}=10.654, \mathrm{t}=7.947$, and $\mathrm{t}=3.134$, respectively; all $P<0.05$ ) (Figure $4 \mathrm{~A}$ ). 
Table 1: Relationship between SOCS3 expression and clinicopathological features of CRC

\section{Clinicopathologic factors}

$\boldsymbol{n}$

\begin{tabular}{|c|c|c|c|}
\hline \multicolumn{4}{|c|}{ SOCS3 } \\
\hline+ & - & $\chi^{2}$ value & $P$ \\
\hline
\end{tabular}

Gender

Men

Women 28

Age (years)

$\leq 40$

$>40$

8

$8 \quad 20$

Tumor size

$\leq 3 \mathrm{~cm}$

$n>3 \mathrm{~cm}$

$\begin{array}{lll}14 & 4 & 10 \\ 26 & 9 & 17\end{array}$

0.001

$>0.05$

Differentiation degree

Well/moderately differentiated

Poorly differentiated

Depth of invasion

Mucosa or superficial muscular layer

Deep muscular layer or whole layer

Lymph node metastasis

Yes

No

$\begin{array}{ll}21 & 10 \\ 19 & 3\end{array}$

11

16

$4.607<0.05$

TNM stage

I+II

III+IV

$\begin{array}{ll}17 & 9 \\ 23 & 4\end{array}$

8

19

$5.631<0.05$

\section{Effect of high SOCS3 expression on the invasion capability of CRC cells by transwell method}

After routine culture of the cells in transwell plates for $24 \mathrm{~h}$, the 8348 SOCS3 cells had significantly lower invasion capability than the controls (Figure 4C-4D). Being counted in each group in four different visual fields, the number of migrating cells in the 8348SOCS3 group was significantly lower than that in the 8348 plv group $(\mathrm{t}=$ 9.590, $P<0.05$ ) (Figure 4B).

\section{The effect of high SOCS3 expression on the invasion and metastasis of CRC in vivo}

\section{Gross observation}

SOCS3 overexpression inhibited the ability of the 8348 cells to grow as tumor xenografts in nude mice, as shown in Figure 5A-5B. Subcutaneous xenografts in nude mice being weighed 28 days after injection, the tumor weight in the 8348SOCS3 group was significantly lower than that in the 8348plv group $(\mathrm{t}=2.802, P<0.05)$ (Figure 5C).

$25 \quad 4 \quad 21$

1596

6

6.389

$<0.05$

$18 \quad 10 \quad 8$

$\begin{array}{lll}22 & 3 & 19\end{array}$
6.134
$<0.05$

\section{Difference in the number of cancer cells in tumor xenografts by HE staining}

Compared with the 8348plv group, the number of cancer cells was significantly decreased in the 8348SOCS3 group, together with a marked reduction in the number of mitotic Figures and in the degree of tumor necrosis (Figure 6A-B).

Expression of SOCS3 and G protein-coupled receptor family C group 5 member A (GPRC5A) in subcutaneous tumor xenografts by immunohistochemical detection

Positive expression of SOCS3 and GPRC5A was mainly located in the cytoplasm. The level of SOCS3 expression was significantly higher in the 8348SOCS3 group $(76.8 \pm 6.2 \%)$ than in the 8348 plv group $(35.4 \pm$ $4.7 \%, P<0.05$ ) (Figure 6C-6D). GPRC5A expression was significantly higher in the $8348 \mathrm{SOCS} 3$ group $(80.2 \pm$ $6.8 \%)$ than in the 8348 plv group $(38.1 \pm 5.3 \%, P<0.05)$ (Figure 6E-6F). Therefore, GPRC5A might partially mediate its anticancer effect by stabilizing SOCS3 and suppressing the STAT3 signaling pathway. 
Table 2: Gene expression profile of 8348 SOCS cells

\begin{tabular}{|c|c|c|c|}
\hline \multicolumn{2}{|c|}{ Up regulated gene } & \multicolumn{2}{|c|}{ Down regulated gene } \\
\hline Gene name & Ratio & Gene name & Ratio \\
\hline SELENBP1 & 2.69 & SLC25A3 & 0.60 \\
\hline MAGED1 & 2.45 & VEGFA & 0.60 \\
\hline BASP1 & 2.44 & KLF9 & 0.60 \\
\hline SPARC & 2.41 & EFNB2 & 0.59 \\
\hline FZD2 & 2.33 & CPEB2 & 0.59 \\
\hline CTGF & 2.32 & DDX3X & 0.60 \\
\hline SOX4 & 2.29 & $\mathrm{ABCC} 5$ & 0.59 \\
\hline FTL & 2.28 & SMC4 & 0.59 \\
\hline ATP1B1 & 2.24 & PTBP2 & 0.59 \\
\hline CSRP2 & 2.24 & UBC & 0.58 \\
\hline MCM3 & 2.24 & SLC39A10 & 0.58 \\
\hline TRIB2 & 2.23 & CCNL1 & 0.58 \\
\hline CCDC80 & 2.23 & PKM & 0.58 \\
\hline NFKBIA & 2.22 & MAT2A & 0.58 \\
\hline GLUD1 & 2.22 & RSRC2 & 0.57 \\
\hline SCARNA14 & 2.22 & PTBP2 & 0.56 \\
\hline GRINA & 2.21 & ZBTB20 & 0.56 \\
\hline 4-Sep & 2.21 & PNISR & 0.53 \\
\hline AP2M1 & 2.21 & MEF2A & 0.60 \\
\hline COL1A2 & 2.21 & PHIP & 0.61 \\
\hline TMSB10 & 2.20 & UCHL5 & 0.64 \\
\hline RBFOX2 & 2.19 & GSK3B & 0.63 \\
\hline CTNNB1 & 2.19 & TAOK1 & 0.64 \\
\hline TPM1 & 2.19 & ZBTB44 & 0.64 \\
\hline GALK1 & 2.19 & RASA1 & 0.64 \\
\hline SSBP3 & 2.18 & ATRX & 0.63 \\
\hline GPX1 & 2.18 & DDX3X & 0.63 \\
\hline BAG6 & 2.17 & PPP4R2 & 0.63 \\
\hline CD248 & 1.13 & ATRX & 0.63 \\
\hline CDH5 & 1.12 & GSK3B & 0.62 \\
\hline
\end{tabular}

The top 60 up- or down-regulated transcripts of SOCS3-8348 cells

Whole-genome expression analysis of the mechanism by which SOCS3 inhibited the growth of CRC cells

Effect of SOCS3 overexpression on the expression of relevant genes

Human genome arrays were used to compare gene expression between 8348SCOS3 and 8348plv cells. We found differential expressions of 369 genes in $8348 \mathrm{SCOS} 3$ cells versus control cells, among which 193 genes (e.g. SELENBP1, MEGED1, and SOX4) showed significantly increased expression and 176 genes (e.g. VEGFA, SMC4, and $A B C C 5$ ) showed significantly decreased expression (Table 2).

Effect of SOCS3 overexpression on signaling pathways

Bioinformatic analysis further demonstrated that high SOCS3 expression might affect multiple signaling pathways in CRC including the TGF- $\beta$ /Smads signaling

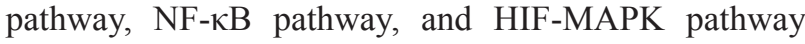


(Figure 7). To investigate the TGF- $\beta /$ Smads signaling pathway we used qRT-PCR to detect the expression of TGF- $\beta 1$ and Smad4 in 8348SCOS3 and 8348plv cells and found that TGF- $\beta 1$ expression was significantly decreased $(P<0.05)$ and Smad4 expression was significantly increased $(P<0.05)$ in 8348 SOCS3 cells (Figure 8A-8B).

\section{DISCUSSION}

SOCS3 is a member of the suppressor of cytokine signaling family and also an important inhibitor of the JAK2/STAT3 signaling pathway. The SOCS3 gene is located on human chromosome 17q25.3 and encodes a protein with molecular weight of $24.7 \mathrm{kDa}$. The SOCS3 protein consists of 225 amino acids that can be divided into an $\mathrm{N}$-terminal domain, a central $\mathrm{SH} 2$ domain, and a C-terminal SOCS box. The kinase inhibitory region (KIR) and the central SH2 domain are key domains through which SOCS3 protein exerts its activity. The C-terminal SOCS box functions like an adaptor and can regulate the normal physiological level of SOCS3 inside cells by controlling the degradation of SOCS3 protein. Research has shown that a decrease or loss of SOCS3 expression may be one of the most important causes of malignancies [15]. Gaballah et al. [16] detected SOCS3 expression in highly malignant bladder cancer, low-grade bladder cancer, and normal bladder tissue using RT-PCR and found that SOCS3 expression was significantly lower in highly malignant bladder cancer than in low-grade bladder cancer and normal bladder tissue, suggesting that decreased SOCS3 expression might be associated with the development of bladder cancer. Chen et al. [17] found that the methylation rate of SOCS3 in human endometrial cancer was $88.3 \%$, which was significantly higher than that in complex endometrial hyperplasia $(53.3 \%)$ and atypical hyperplasia (54.2\%). It was speculated that methylation-silenced SOCS3 plays a key role in the pathogenesis of endometrial carcinoma.

To date, few studies have explored the relationship between SOCS3 and the clinicopathological features of $\mathrm{CRC}$, and its mechanisms of action remain unclear. In our current study, we demonstrated that SOCS3 expression was significantly lower in CRC than in normal colorectal tissues by immunohistochemical methods and qRTPCR, which suggested that decreased SOCS3 expression might be involved in the occurrence and progression of CRC. Further analysis showed that SOCS3 expression was correlated with tumor differentiation degree, depth of invasion, TNM stage, and lymph node metastasis, implying that low SOCS3 expression was associated with other aggressive biological behaviors such as invasion and metastasis. Thus, SOCS3 may be a useful biological indicator for assessing the biological behaviors of CRC and predicting prognosis.

To further study the relationship between SOCS3 level and the biological behaviors of CRC cells such as proliferation, adhesion and invasion, we constructed a CRC cell line that was transfected with SOCS3 vector and showed high expression of SOCS3 (8348SOCS3 cells). Then we compared them with the parental cells transfected with empty vector (8348plv cells). We first examined the effect of SOCS3 overexpression on the growth and proliferation of CRC cells using the CCK8 method. It was observed that the proliferation rate of

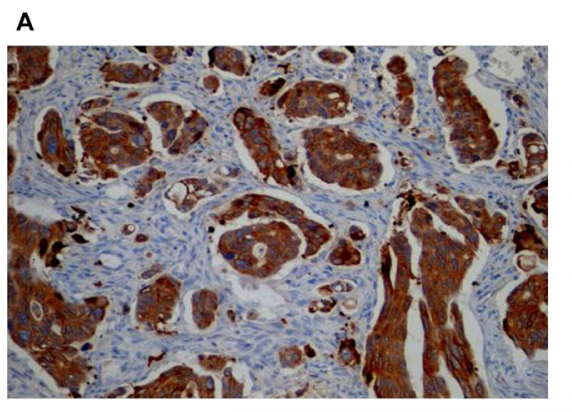

B
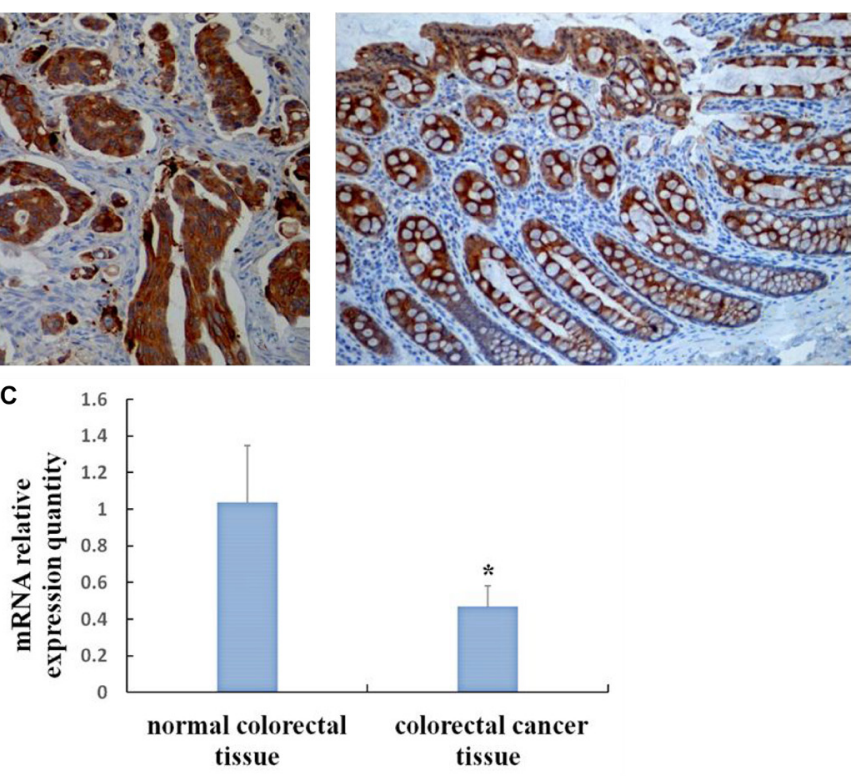

Figure 1: Expression of SOCS3 in CRC and normal colorectal tissue by immunohistochemical method and qRT-PCR. (A) Positive expression of SOCS3 protein in Colorectal cancer tissues (SP, $\times 200)$; (B) Positive expression of SOCS3 protein in Normal colorectal tissues (SP, $\times 200$ ); (C) SOCS3 mRNA levels in colorectal cancer tissue and normal colorectal tissue. ${ }^{*} P<0.01$, compared with normal colorectal tissue. 
8348SOCS3 cells was remarkably decreased compared with controls. In addition, we applied the transwell method to investigate the effect of SOCS3 overexpression on the invasion capability of CRC cells. It was found that the number of migrating cells in 8348 SOCS3 group was significantly smaller than that in 8348 plv group after 24 hours of incubation. These results were consistent with the findings of Kathryn et al. [18], which suggested that SOCS3 overexpression could suppress the proliferation and invasion of CRC cells. Therefore artificially inducing SOCS3 expression might provide a new approach to therapy for CRC.

To validate the cytological findings, we performed an in vivo experimental study in which 8348SOCS3 cells and 8348 plv cells were separately grafted into the subcutaneous tissues of nude mice. After 28 days, the weight of the subcutaneous tumor xenografts in the 8348 SOCS3 group was significantly lower than that in the 8348 plv group. In addition, compared with the 8348plv group, the number of cancer cells in tumor xenografts was significantly decreased in the 8348SOCS3 group, together with a marked decrease in mitotic figures and in the degree of tumor necrosis. These results suggested that SOCS3 overexpression might inhibit the occurrence and progression of CRC.

GPRC5A, also known as Retinoic acid-induced protein 3 (RAI3), is a retinoic acid-inducible gene. GPRC5A gene locus is at $12 \mathrm{p} 13$. LOH of chromosome $12 \mathrm{p}$ was found to frequently occur in NSCLCs. It is a newly discovered tumor suppressor gene [19-21]. In GPRC5A-/- mouse tracheal epithelial, the STAT3 signaling was significantly increased [22]. YL Chen
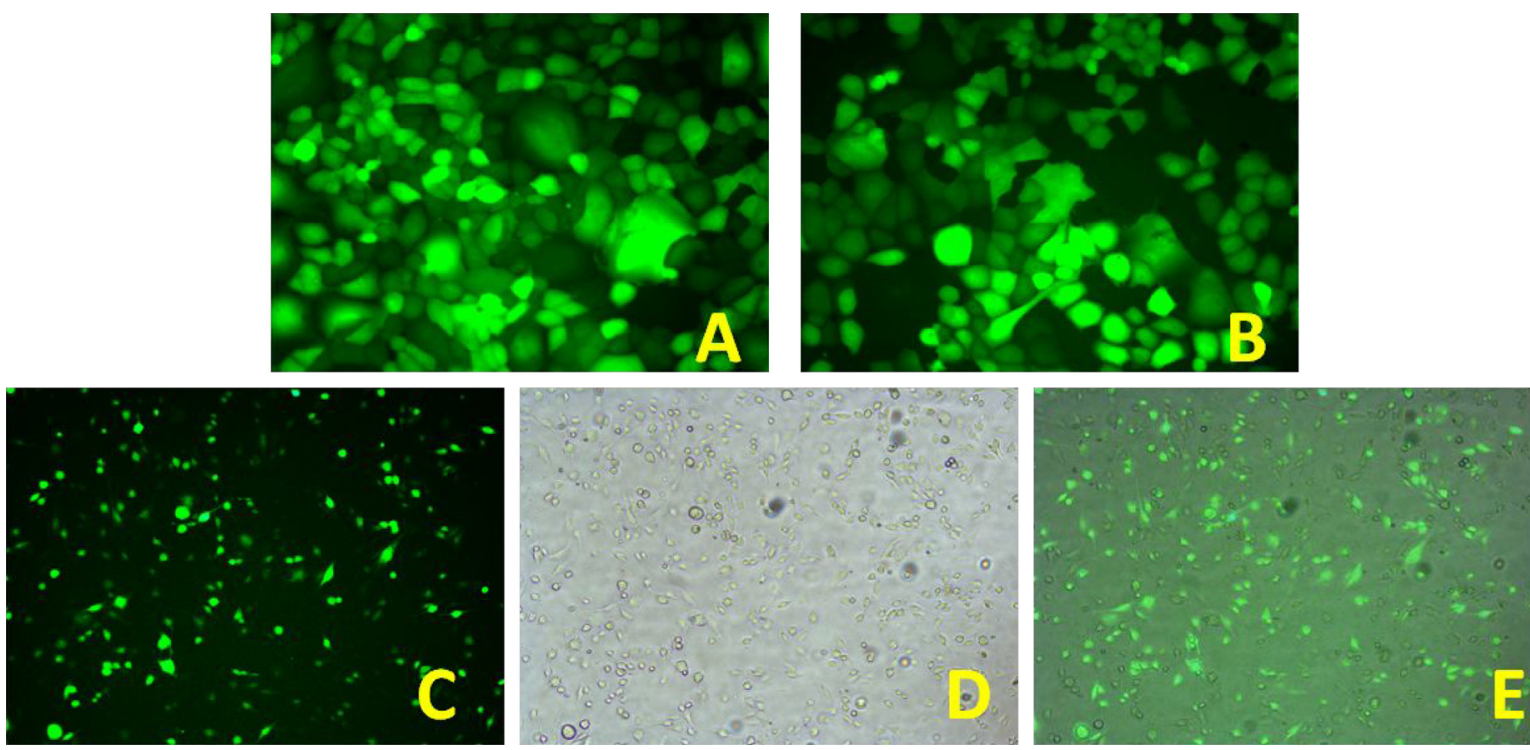

Figure 2: (A) 293 packaging cells that secrete lentivirus particles $(\times 40)$; (B) 293 cells observed under a fluorescence microscope $(\times 40)$. (C) GFP-positive 8348 cells after sorting $(\times 10)$; (D) 8348 cells under a light microscope after sorting $(\times 10)$; (E) Merged image of C and D.

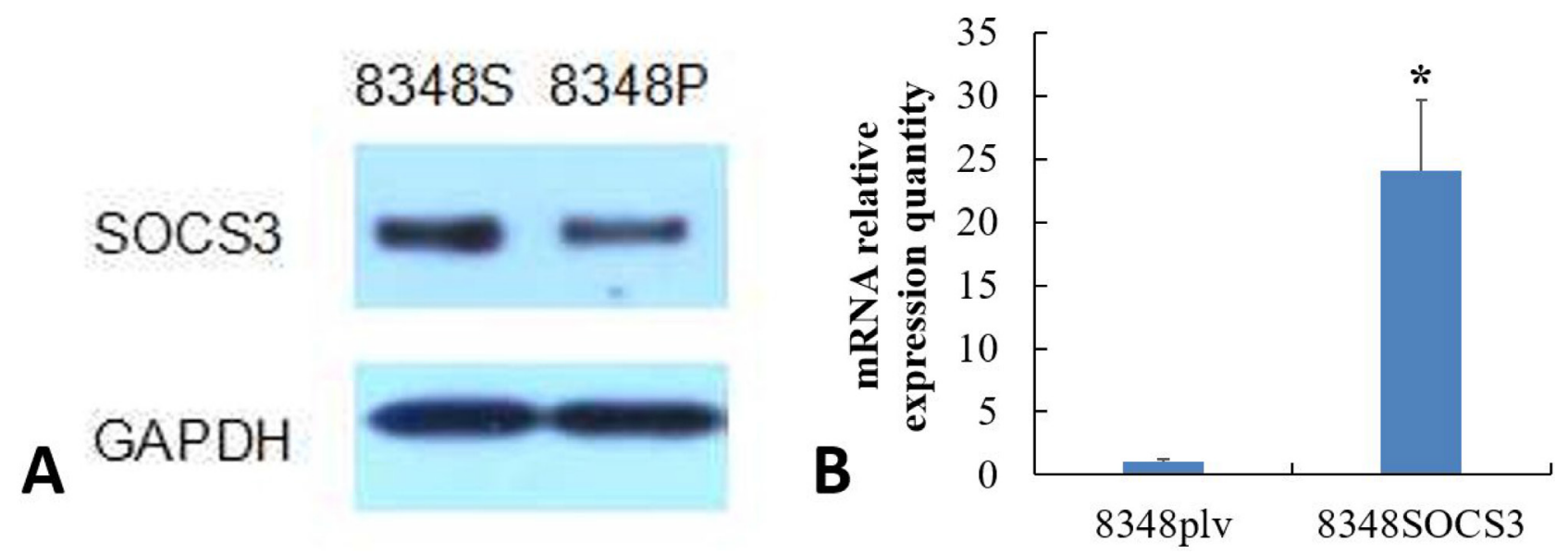

Figure 3: (A) SOCS3 protein expressions in 8348 SOCS3 and 8348 plv cells by western blotting. (B) SOCS3 mRNA expression in 8348SOCS3 and 8348plv cells by qRT-PCR. ${ }^{*} P<0.01$, compared with 8348 plv cells. 
et al [22] indicate that the lower SOCS3 level in the GPRC5A-/- cells may be responsible for the persistent activation of STAT3, which regulate cell proliferation, differentiation, survival, invasion and lead to the development of tumors. Using immunohistochemical methods, we showed that GPRC5A expression was significantly higher in subcutaneous tumor xenografts of the 8348 SOCS3 group compared with the $8348 \mathrm{plv}$ group. We therefore speculated that SOCS3 might at least partially exert its anticancer effect by stabilizing GPRC5A and suppressing the STAT3 signaling pathway.

At present, the mechanism by which SOCS3 influences the occurrence and development of CRC is still not clear. As one of the most potent suppressor proteins in negative regulation of the JAK/STAT signaling pathway, SOCS3 may competitively bind to the phosphorylated Tyr site in the cytoplasmic region of cytokine receptors through its $\mathrm{SH} 2$ domain, which mimics the transcription factor STAT, and thus prevent activation of STAT signaling. In addition, SOCS3 can bind to JAK1, JAK2, and TYK2 and directly suppress the catalytic activities of these kinases, thus it can inhibit oncogenes and prevent the occurrence and development of tumors $[9,23]$. In the current study, we used a human genome array to compare gene expression between 8348SOCS3 cells and 8348plv cells. We found that the expression of 369 genes was remarkably changed in 8348SOCS3 cells. Of
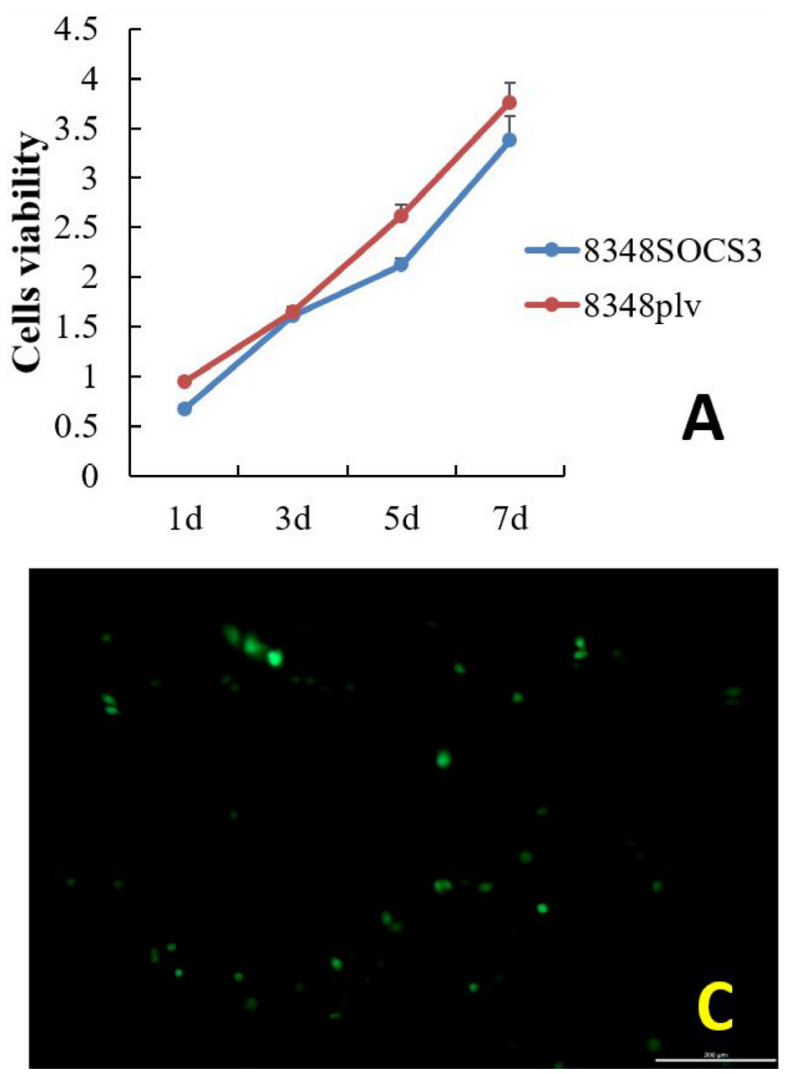

these, 193 genes (e.g., SELENBP1, MEGED1, and SOX4) showed significantly increased expression and 176 genes (e.g., VEGFA, SMC4, and ABCC5) showed significantly decreased expression. Most of these genes were involved in tumorigenesis. So we speculated that SOCS3 might inhibit the occurrence, invasion, and metastasis of CRC by inducing expression of tumor suppressor genes or by suppressing the expression of oncogenes. In addition, bioinformatic analysis further demonstrated that high SOCS3 expression could affect multiple signaling pathways in CRC including the TGF- $\beta$ / Smads signaling pathway, NF- $\mathrm{B}$ pathway, and HIF-MAPK pathway. The TGF- $\beta /$ Smads signaling pathway is closely associated with the occurrence and development of tumors in the human body. Abnormality of any of its components can cause a signaling disorder and thus lead to tumorigenesis [24, $25]$. By qRT-PCR we showed that TGF- $\beta 1$ expression was significantly decreased $(P<0.05)$ and Smad4 expression was significantly increased $(P<0.05)$ in 8348 SOCS3 cells compared with 8348 plv cells. Therefore, SOCS3 may exert its effect by inhibiting the TGF- $\beta 1 /$ Smads signaling pathway. TGF- $\beta 1$, as a fundamental mediator of ECM, plays a critical role in the epithelial-mesenchymal transition (EMT) process by a Smad-dependent pathway. TGF- $\beta 1$ promotes EMT by which epithelial cells lose their orientation and cell-cell contact, and acquire migratory and invasive properties of cells, resulting in tumor metastasis [26].
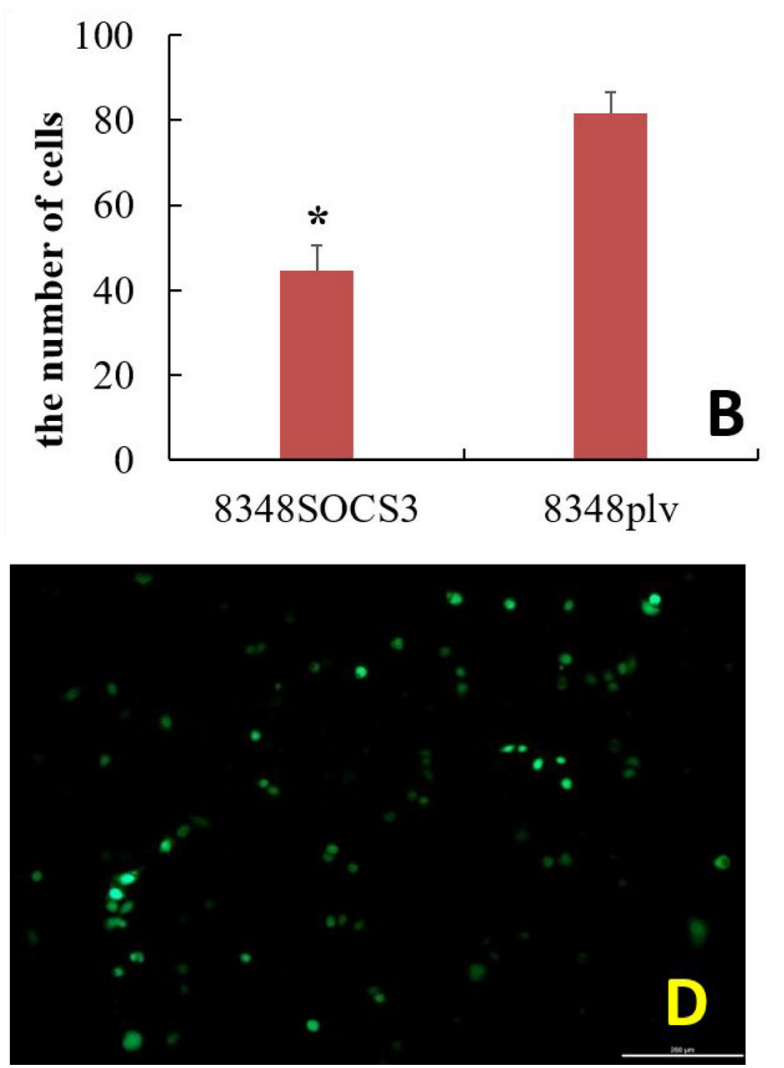

Figure 4: Effect of high SOCS3 expression on the growth, proliferation and invasion capability of CRC cells. (A) Growth curve of colorectal cancer cells with and without transfection with SOCS3. (B) The number of cells penetrating the membrane was significantly lower in the 8348SOCS3 group than in the 8348plv group $\left({ }^{*} P<0.05\right)$. Fluorescence microscopy of 8348 cells after culture in transwell chamber for $24 \mathrm{~h}$. (C) 8348 SOCS3 cells; (D) 8348plv cells. 
In summary, low expression of SOCS3 is closely associated with the occurrence, progression, invasion, metastasis, and other biological behaviors of CRC. Therefore, it may be a useful indicator for assessing the biological behavior of CRC and predicting prognosis. Artificially induced SOCS3 expression may provide a new target for gene therapy of CRC. High SOCS3 expression may exert its antitumor effect by affecting multiple signaling pathways, including the TGF- $\beta /$ Smads signaling pathway, NF-кB pathway, and HIF-MAPK pathway in CRC. However, its specific mechanisms require further investigation.

\section{MATERIALS AND METHODS}

\section{Ethics statement}

The study was approved by the Clinical Research Ethical Committee of PLA ARMY GENERAL HOSPITAL and informed written consent was obtained from all subjects.

\section{Detection of SOCS3 expression in CRC}

\section{Sample collection}

Surgically resected CRC specimens were harvested from 40 patients aged 33-70 years (mean: 53 years) who were treated in Department of General Surgery, PLA Army General Hospital from January 1, 2015 to December 30, 2015. Adjacent normal colorectal mucosa was used as the controls. Two blocks of CRC tissue and adjacent normal colorectal mucosa (sized approximately $1.0 \times 1.0 \times 1.0$ $\mathrm{cm}$ ) were harvested immediately after the surgery. One block was quickly fixed in 10\% neutral formaldehyde, and the other was stored at $-80^{\circ} \mathrm{C}$ for qRT-PCR.

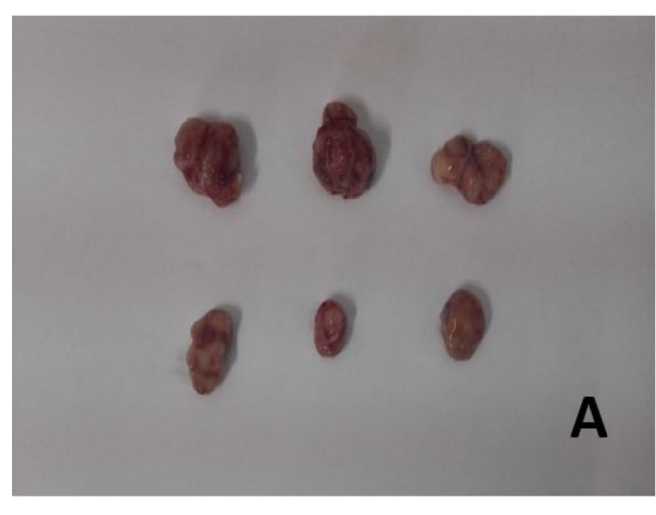

\section{Immunohistochemical determination}

The SP (streptavidin perosidase) immunohistochemical method was applied. The rabbit anti-human SOCS3 polyclonal antibody was purchased from Santa Cruz Biotechnology (Santa Cruz, CA, USA; 1:100 dilution). For each slice, five high-power fields were randomly selected for analysis. A comprehensive score was calculated according to the staining intensity and the proportion of positive cells among the total number of tumor cells. [14] Scoring for staining intensity was as follows: 0, no specific staining; 1, light yellow; 2, brownish yellow; and 3, brown. Scoring for the proportion of the positive cells was as follows: 0 , less than $5 \% ; 1$, $5-25 \% ; 2,26-50 \%$; and 3 , greater than 50\%. The final score was obtained by multiplying these two scores; a value of $0-1$ was regarded as negative and a score $\geq 2$ as positive $(+)$.

\section{qRT-PCR}

Total RNA was isolated from CRC tissue and normal colorectal mucosa tissue with Trizol reagent (Invitrogen Life Technologies, Paisley, UK) according to the manufacturer's instructions and reverse transcribed. qRT-PCR was performed with Universal SYBR Green PCRMaster Mix (TaKaRa) using specific primers with the following sequences: SOCS3 forward 5'-ATCCTGGTGACATGCTCCTC-3', reverse 5'-CAAATGTTGCTTCCCCCTTA-3'; $\beta$-actin forward 5'-GATCCACATCTGCTGGAAGG-3', reverse 5'-AAGTGTGACGTTGACATCCG-3'. The reaction conditions were as follows: $94^{\circ} \mathrm{C}$ for $5 \mathrm{~min}$; $94^{\circ} \mathrm{C}$ for 30 $\mathrm{s}, 56^{\circ} \mathrm{C}$ for $30 \mathrm{~s}$, and $72^{\circ} \mathrm{C}$ for $40 \mathrm{~s}(40$ cycles $)$; and $72^{\circ} \mathrm{C}$ for $10 \mathrm{~min}$. Relative quantification was determined using the comparative CT method.

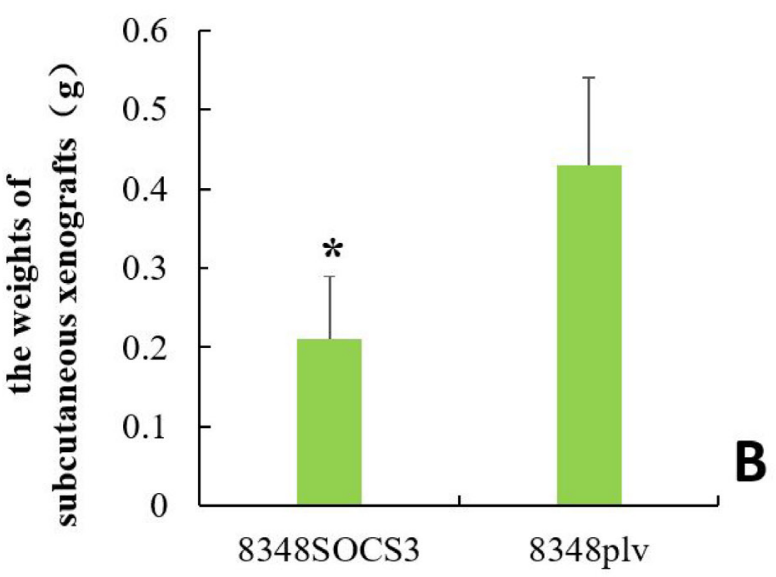

Figure 5: Tumor xenografts in nude mice. (A) Tumor formation by different cells in nude mice; (B) Comparison of the weights of subcutaneous xenografts between 8348SOCS3 group and 8348plv group $\left({ }^{*} P<0.05\right)$. 


\section{Effect of SOCS3 overexpression on the biological features of CRC cells}

\section{Cell culture}

8348 cells and 293FT cells were purchased from the Peking Union Medical College Hospital cell bank. The 8348 cells were cultured in RPMI-1640 medium (Sigma, Santa Clara, CA, USA) containing $10 \%$ fetal bovine serum (GIBCO BRL, Gaithersburg, MD, USA) and 293FT cells were cultured in DMEM complete medium high-glucose supplemented (Sigma) with 10\% fetal bovine serum under standard conditions $\left(37^{\circ} \mathrm{C}, 5 \% \mathrm{CO}_{2}\right.$, and saturated humidity).

\section{Cloning of human SOCS3 gene}

Total RNA of 8348 cells was extracted by the conventional Trizol method for cDNA synthesis. Cloning
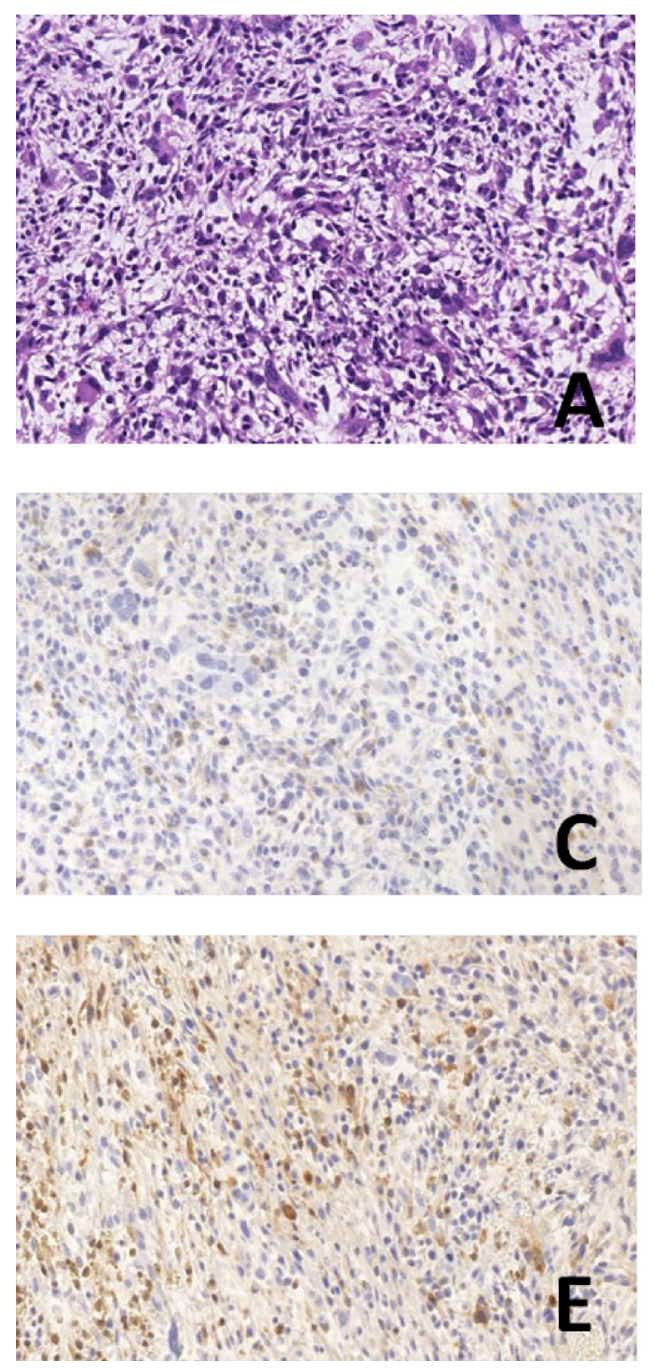

of the SOCS3 gene was performed by a PCR-based method. The PCR product was characterized by $1 \%$ agarose gel electrophoresis and subjected to restriction endonuclease digestion. The digestion products were separated by agarose gel electrophoresis and SOCS3 gene product and vector fragments were separately recovered using a gel recovery kit (Qiagen company). The recovery products were mixed in a molar ratio of $3: 1$ and incubated with T4 DNA ligase at $16^{\circ} \mathrm{C}$ overnight. The resultant SOCS3 recombinant plasmid was transferred into Escherichia coli DH5 $\alpha$ cells for amplification. The transformed bacteria were plated on LB agar plates containing ampicillin and cultured overnight.

\section{Clone identification}

A single colony was inoculated in LB medium containing ampicillin and shaked overnight at $37^{\circ} \mathrm{C}$. Plasmids were extracted for screening and identification.
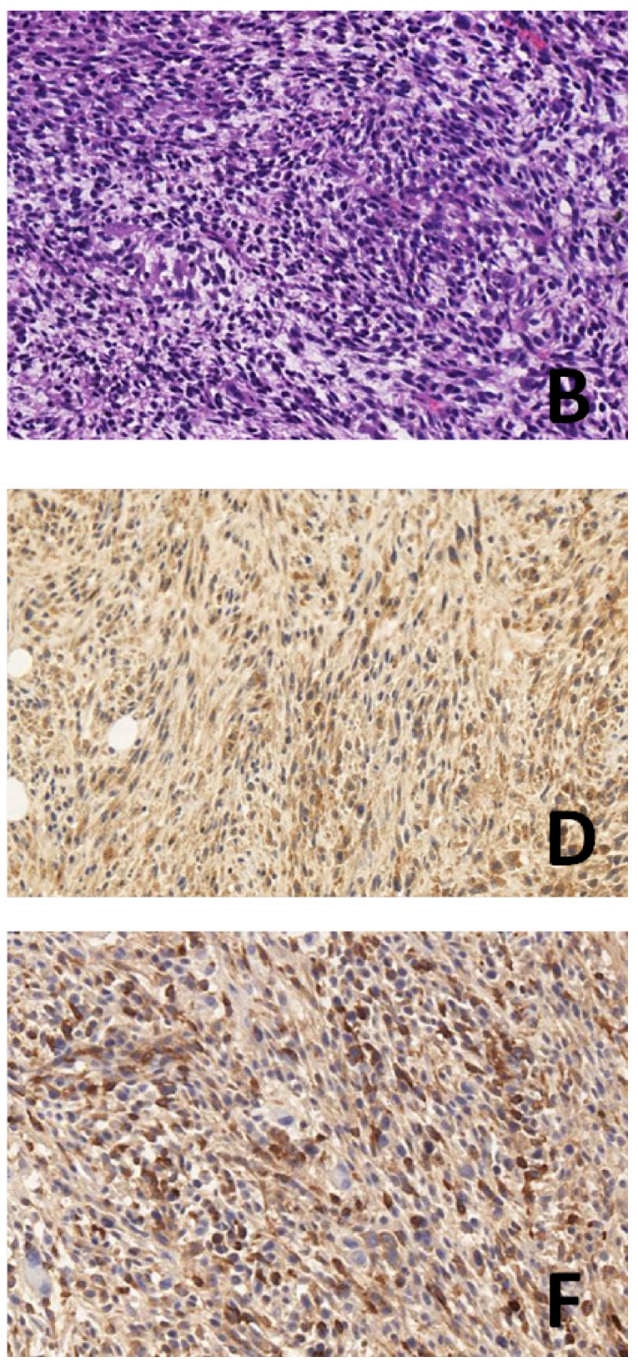

Figure 6: HE and immunohistochemical detection of subcutaneous tumor xenografts in nude mice $(\times 400)$. (A) 8348SOCS3 group in HE; (B) 8348plv group in HE; (C) SOCS3 expression in 8348plv group; (D) SOCS3 expression 8348SOCS3 group; (E) GPRC5A expression in 8348plv group; (F) GPRC5A expression in 8348SOCS3 group. 
The positive bacterial suspension was sent to Sangon Co., Ltd (Shanghai, China) for sequencing. The sequencing results were compared with published databank proteins to confirm the cloned target gene fragments. The suspension containing the correct clone was amplified and recombinant plasmids were extracted and stored at $-20^{\circ} \mathrm{C}$ for further use.

\section{Packaging of lentivirus}

The expression vector for the constructed SOCS3 lentivirus, together with the packaging plasmids and envelope plasmids (Invitrogen), was mixed with lipofectamine 2000 in serum-free high-glucose DMEM medium and incubated at room temperature for 20 min to form the lipofectamine 2000/DNA complex. After trypsin digestion of 293FT cells, approximately $6 \times 10^{6}$ cells were harvested and resuspended in $5 \mathrm{ml}$ of growth medium. After addition of the lipofectamine 2000/DNA complex and thorough mixing, the cells were transferred to a $10-\mathrm{cm}$ cell culture dish containing $5 \mathrm{ml}$ of growth medium and inoculated overnight in a $\mathrm{CO}_{2}$ incubator at $37^{\circ} \mathrm{C}$. The next day, the medium was changed to complete medium containing $1 \mathrm{mmol} / \mathrm{L}$ sodium pyruvate. At 48 and $72 \mathrm{~h}$ after transfection, the supernatant was collected and centrifuged at 3,000 rpm for $15 \mathrm{~min}$ to remove cell debris before storage at $-80^{\circ} \mathrm{C}$ for further use.

\section{Cell transfection and flow cytometry}

The 8348 cells were collected by centrifugation. Meanwhile, $2 \mathrm{ml}$ of virus supernatant was added to polybrene at a final concentration of $6 \mathrm{mg} / \mathrm{L}$. Cells were resuspended in this solution and incubated in a 96-well plate overnight at a density of $2.5 \times 10^{5}$ cells $/ \mathrm{ml}$. The next day, the medium was changed and the cells were cultured for a further $72 \mathrm{~h}$. At 48 and $72 \mathrm{~h}$ after transfection, the cell transfection efficiency was observed under a fluorescence microscope. The transfected cells were collected for flow cytometry to obtain 8348 cells with high green fluorescent protein (GFP) expression.

\section{Detection of $\mathrm{SCOC} 3$ expression after transfection by western blotting and qRT-PCR}

CRC cells with SOCS3 transfection (8348SOCS3) and without SOCS3 transfection (8348plv) were harvested. Total protein was extracted and the protein concentration was measured with BCA Protein Quantification Kit (Beijing Kangwei Century Biotechnology Co Ltd). Samples were separated by $15 \%$ SDS-PAGE and transferred to polyvinylidene difluoride (PVDF) membranes using the semi-dry transfer method. Samples were blocked with 5\% skimmed milk powder at room temperature for $2 \mathrm{~h}$, washed with TBST, and incubated with goat anti-human SOC3-3 monoclonal antibody (Santa Cruz Biotechnology) overnight at $4^{\circ} \mathrm{C}$. After washing with TBST, HRP-labeled mouse anti-goat second antibody (Santa Cruz Biotechnology) was added and incubated at room temperature for $1 \mathrm{~h}$ before a final wash with TBST. Autoradiography was conducted using chemiluminescent substrate. GAPDH protein was used as an internal loading control.

Total RNA was extracted from 8348 SOCS3 cells and $8348 \mathrm{plv}$ cells by the Trizol method. After reverse transcription, one sample of cDNA was used for a gradient dilution for generation of a standard curve; PCR was performed simultaneously with the other samples. The standard curve was drawn for relative quantitative analysis.

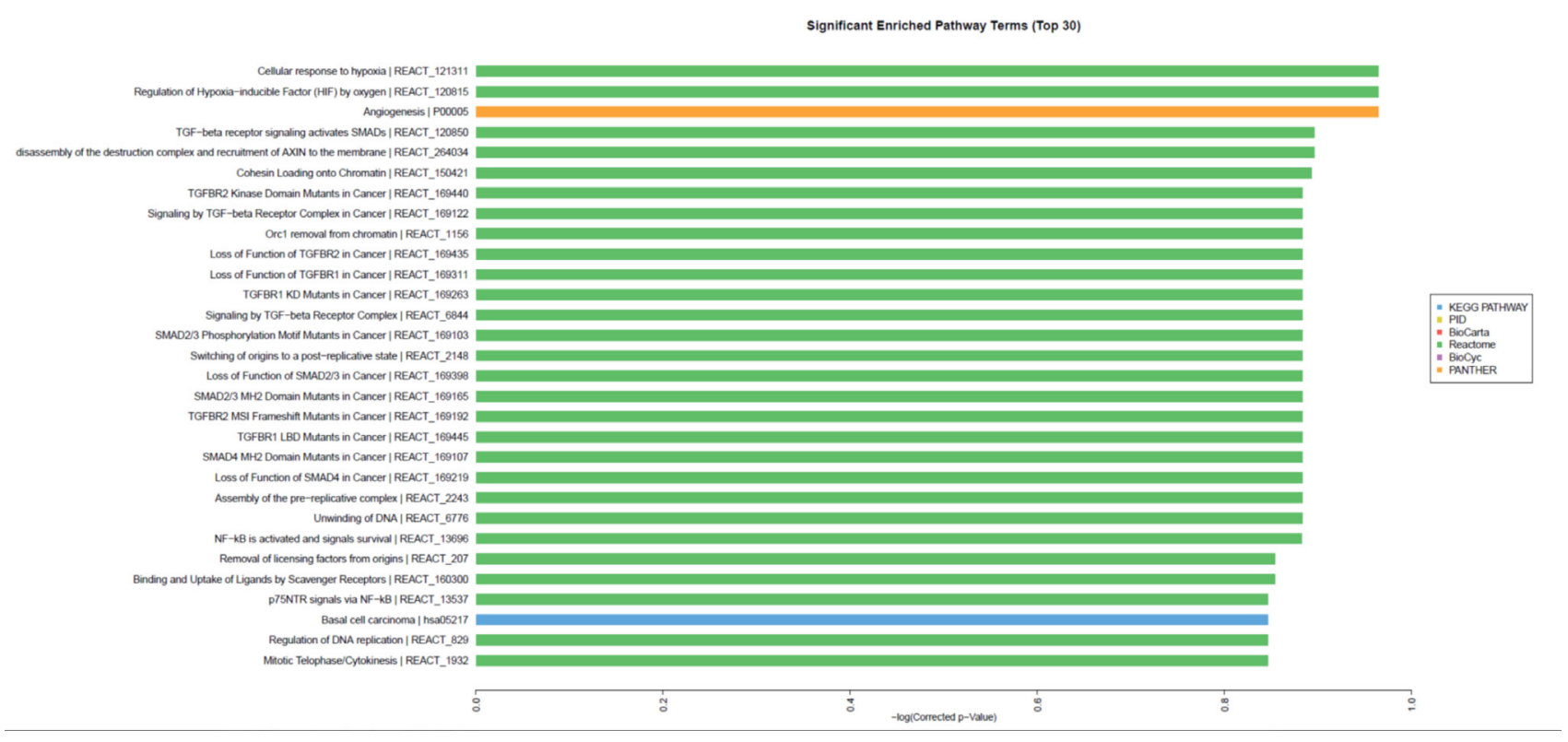

Figure 7: Effect of SOCS3 overexpression on multiple signaling pathways of CRC. 


\section{Effect of SOCS3 overexpression on the growth and proliferation of $\mathrm{CRC}$ cells using the $\mathrm{CCK}-8$ method}

8348SOCS3 cells and 8348plv cells were harvested and rinsed with $5 \mathrm{ml}$ of PBS. Five hundred microliters of trypsin was added to digest the cells, and the digestion was terminated by addition of DMEM with $10 \%$ FBS. Cells were counted and plated in a 96-well plate at a density of $2 \times 10^{4}$ cells $/ \mathrm{ml}$. Four parallel wells were set up with $100 \mu 1$ cellular suspension in each well, and the plate was placed in a $\mathrm{CO}_{2}$ incubator and incubated overnight. At fixed measurement time points $10 \mu 1$ of CCK- 8 agent was added to every well, followed by incubation in a $5 \% \mathrm{CO}_{2}$ incubator at $37^{\circ} \mathrm{C}$ for $2 \mathrm{~h}$. The optical density (OD) of the cells was measured with a detection wavelength of 450 $\mathrm{nm}$. The OD value on the first day was used as the blank control.

\section{Effect of SOCS3 overexpression on the invasion of CRC cells using a transwell method}

Matrigel was added to the upper chamber of the transwell, which was then incubated at $37^{\circ} \mathrm{C}$ for $30-40$ min for gelling. The 8348 SOCS3 and 8348plv cell suspensions were digested and incubated in the upper chamber of the transwell at a density of $5 \times 10^{4}$ cells $/ \mathrm{ml}$ for $24 \mathrm{~h}$. After fixation in 4\% polyformaldehyde for 15 20 min the cells were observed and photographed under a fluorescence microscope.

\section{Effect of high SOCS3 expression on the invasion and metastasis of CRC in vivo}

\section{Gross examination}

The 8348 SOCS3 cells and 8348 plv cells were collected by trypsin digestion. With three nude mice in

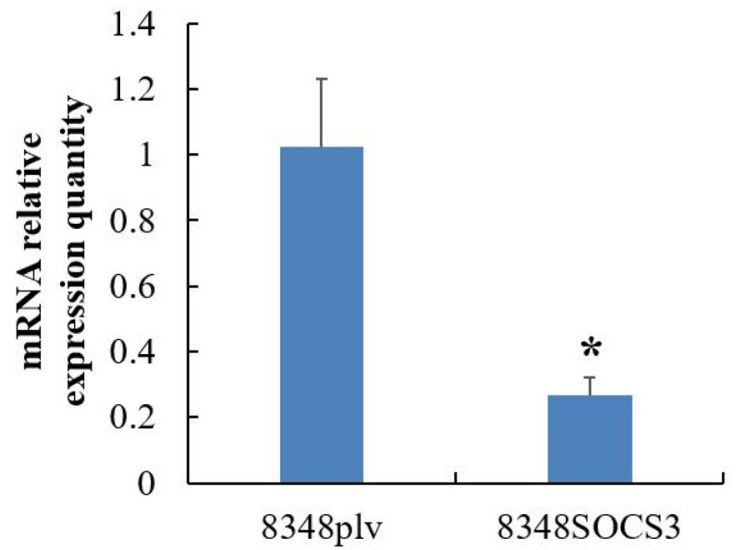

each group, each mouse was designed to receive $1 \times 10^{7}$ cells in a $100-\mu 1$ volume at each of the injection sites. The 3\% pentobarbital sodium was diluted 1:10 with PBS before use. The dose of the anesthetic (after dilution) was $20 \mu \mathrm{L} / \mathrm{g}$ for each mouse. Cells were grafted under the dorsal skin of nude mice. After 28 days, the tumor sizes were measured and photographed.

\section{Detection of SOCS3 and GPRC5A expression by HE staining and immunohistochemistry}

The tumor tissue was cut into tissue blocks sized $0.5 \mathrm{~cm}^{3}$ to produce paraffin-embedded tumor sections. The other steps for immunohistochemical determination were performed as described above. Using known SOCS3- and GPRC5A-positive sections as the positive controls, cells with intracellular brown-yellow particles were regarded as positive. A total of 100 cells were counted in each high-power field $(\times 400)$. The rate of positive cells was calculated as number of positive cells/total number of cells $\times 100 \%$.

\section{Whole-genome expression analysis of the mechanism by which SOCS3 regulates the growth of CRC cells}

Total RNA was extracted from 8348SOCS3 cells and 8348plv cells using Trizol reagent. Genome expression analysis was performed using an Illumina Human HT-12 v4 BeadChip (Illumina, San Diego, CA, USA) at the Beijing Qian Zhao Xing Ye Biological Technology Co., Ltd. (Beijing, China). The beadchips were scanned on the Illumina Bead Array 500GX Reader using Illumina BeadScan image data acquisition software. Illumina BeadStudio software was used for preliminary data analysis. The preliminary data were normalized using sample averages; the sample intensities were scaled by a factor equal to the ratio of average intensity of a sample to

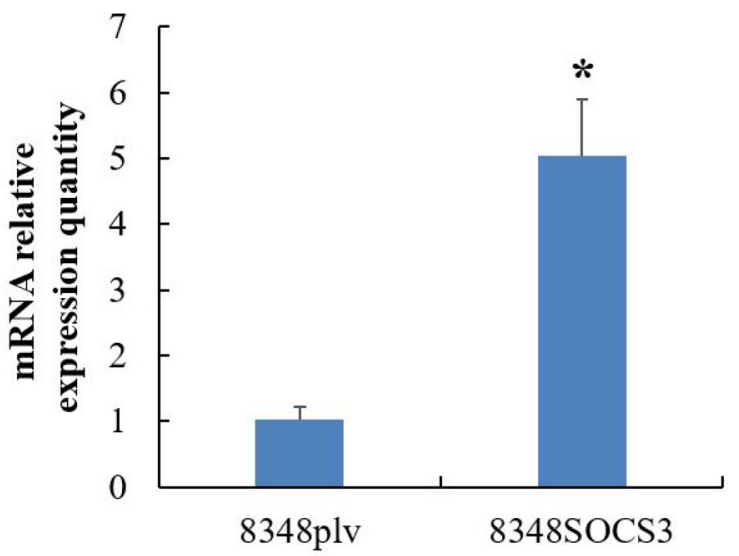

Figure 8: Expression of TGF- $\beta 1$ and Smad4 in TGF- $\beta$ /Smads signaling pathway in 8348SCOS3 and 8348plv cells by real-time RT-PCR. (A) TGF- $\beta 1$ expression was significantly decreased in $8348 \mathrm{~S}$ cells; (B) Smad4 expression was significantly increased in $8348 \mathrm{~S}$ cells. 
the average intensity of the given sample [8]. 8348SOCS3 cells and 8348plv cells were regarded as the given sample. Each sample was repeated three times. An Illumina custom algorithm was used to compare 8348 SOCS3 cells with $8348 \mathrm{plv}$ cells. A difference score for a probe (diff score) indicates differential gene expression between the two groups. For each gene, the diff scores of corresponding probes were averaged. The results were validated using qRT-PCR.

\section{Statistical analysis}

All data analysis was performed using SPSS 13.0 software (SPSS Inc., Chicago, IL, USA). Measurement data were presented as means \pm SD and analyzed using Student's t test. Comparison of count data was performed using chi square test. $P<0.05$ was considered statistically significant.

\section{ACKNOWLEDGMENTS}

We thank professor Shi-Yong Li for generously providing 8348 cells. We also grateful to Dr. Yu-Xiao Liu, Qiang Yuan and Miss Hui-Yun Cai for helping recruiting specimens and providing experimental support for this study.

\section{CONFLICTS OF INTEREST}

The authors declare no conflicts of interest.

\section{REFERENCES}

1. Huang CJ, Lee CL, Yang SH, Chien CC, Huang CC, Yang $\mathrm{RN}$, Chang CC. Upregulation of the growth arrest-specific-2 in recurrent colorectal cancers, and its susceptibility to chemotherapy in a model cell system. Biochim Biophys Acta. 2016; 1862:1345-1353.

2. Wang N, Wang L, Yang H, Zhang HQ, Lan B, He X, Jin TB, Kang LL, Chen C. Multiple genetic variants are associated with colorectal cancer risk in the Han Chinese population. Eur J Cancer Prev. 2015; 24:1-5.

3. Binefa G, Rodriguez-Moranta F, Teule A, Medina-Hayas M. Colorectal cancer: from prevention to personalized medicine. World J Gastroenterol. 2014; 20:6786-6808.

4. Konda K, Konishi K, Yamochi T, Ito YM, Nozawa H, Tojo M, Shinmura K, Kogo M, Katagiri A, Kubota Y, Muramoto T, Yano Y, Kobayashi Y, et al. Distinct molecular features of different macroscopic subtypes of colorectal neoplasms. PLoS One. 2014; 9:e103822.

5. Li G, Xu J, Wang Z, Yuan Y, Li Y, Cai S, He Y. Low expression of SOCS-1 and SOCS-3 is a poor prognostic indicator for gastric cancer patients. J Cancer Res Clin Oncol. 2015; 141:443-452.

6. Stofas A, Levidou G, Piperi C, Adamopoulos C, Dalagiorgou G, Bamias A, Karadimou A, Lainakis GA,
Papadoukakis S, Stravodimos K, Dimopoulos MA, Patsouris E, Gakiopoulou H, et al. The role of CXCchemokine receptor CXCR2 and suppressor of cytokine signaling-3 (SOCS-3) in renal cell carcinoma. BMC Cancer. 2014; 14:149-165.

7. Zhang MY, Fung TK, Chen FY, Chim CS. Methylation profiling of SOCS1, SOCS2, SOCS3, CISH and SHP1 in Philadelphia-negative myeloproliferative neoplasm. J Cell Mol Med. 2013; 17:1282-1290.

8. Liu YX, Dong X, Gong F, Su N, Li SB, Zhang HT, Liu JL, Xue JH, Ji SP, Zhang ZW. Promotion of Erythropoietic Differentiation in Hematopoietic Stem Cells by SOCS3 Knock-Down. PLoS One. 2015; 10:e135259.

9. Chen YY, Ma ZB, Xu HY, Shi LJ, Li DY, Sun LY, Yin XH, Sang GY, Xu D, Tang YH, Wang X, Li P, Wu F, et al. IL-6/ STAT3/SOCS3 signaling pathway playing a regulatory role in ulcerative colitis carcinogenesis. Int J Clin Exp Med. 2015; 8:12009-12017.

10. Wang Y, Wan M, Zhou Q, Wang H, Wang Z, Zhong X, Zhang L, Tai S, Cui Y. The Prognostic role of SOCS3 and A20 in human cholangiocarcinoma. PLoS One. 2015; 10:ep141165.

11. Rossa CJ, Sommer G, Spolidorio LC, Rosenzweig SA, Watson DK, Kirkwood KL. Loss of expression and function of SOCS3 is an early event in HNSCC: altered subcellular localization as a possible mechanism involved in proliferation, migration and invasion. PLoS One. 2012; 7:e45197.

12. Tomita S, Ishibashi K, Hashimoto K, Sugino T, Yanagida T, Kushida N, Shishido K, Aikawa K, Sato Y, Suzutani T, Yamaguchi O. Suppression of SOCS3 increases susceptibility of renal cell carcinoma to interferon-alpha. Cancer Sci. 2011; 102:57-63.

13. Kneitz B, Krebs M, Kalogirou C, Schubert M, Joniau S, van Poppe H, Lerut E, Kneitz S, Scholz CJ, Strbel P, Gessler M, Riedmiller H, Spahn M. Survival in patients with highrisk prostate cancer patients is predicted by mir-221, which regulates proliferation,apoptosis and invasion of prostate cancer cells by inhibiting IRF2 and S0CS3. Cancer Res. 2014; 74:2591-2603.

14. Zhang Y, Zhang M, Yu F, Lu S, Sun H, Tang H, Peng Z. Karyopherin alpha 2 is a novel prognostic marker and a potential therapeutic target for colon cancer. J Exp Clin Cancer Res. 2015; 34:145-156.

15. Wang J, Zhou H, Han Y, Liu X, Wang M, Wang X, Yin G, Li X, Xiang M. SOCS3 methylation in synergy with Reg3A overexpression promotes cell growth in pancreatic cancer. J Mol Med (Berl). 2014; 92:1257-1269.

16. Gaballah HH, Shafik NM, Wasfy RE, Abou Farha MO. Significance of suppressor of cytokine signaling-3 expression in bladder urothelial carcinoma in relation to proinflammatory cytokines and tumor histopathological grading. Asian Pac J Cancer Prev. 2015; 16:307-314.

17. Chen H, Zhang C, Sheng Y, Yao S, Liu Z, Zhang C, Zhang T. Frequent SOCS3 and 3OST2 promoter methylation and 
their epigenetic regulation in endometrial carcinoma. Am J Cancer Res. 2014; 5:180-190.

18. Hamilton KE, Simmons JG, Ding S, Van Landeghem L, Lund PK. Cytokine induction of tumor necrosis factor receptor 2 is mediated by STAT3 in colon cancer cells. Mol Cancer Res. 2011; 9:1718-1731.

19. Zhong SS, Yin HJ, Liao YL, Yao F, Li Q, Zhang J, Jiao HK, Zhao YX, Xu DL, Liu SL, Song HY, Gao Y, Liu JY, et al. Lung tumor suppressor GPRC5A binds EGFR and Restrains its effector signaling. Cancer Res. 2015; 75:1801-1814.

20. Zhou H, Rigoutsos I. The emerging roles of GPRC5A in diseases. Oncoscience. 2014; 1:765-776. http://doi. org/10.18632/oncoscience. 104.

21. Deng J, Fujimoto J, Ye XF, Men TY, Van Pelt CS, Chen YL, Lin XF, Kadara H, Tao QG, Lotan D, Lotan R. Knockout of the tumor suppressor gene Gprc5a in mice leads to NFkappaB activation in airway epithelium and promotes lung inflammation and tumorigenesis. Cancer Prev Res (Phila). 2010; 3:424-437.

22. Chen Y, Deng J, Fujimoto J, Kadara H, Men T, Lotan D, Lotan R. Gprc5a deletion enhances the transformed phenotype in normal and malignant lung epithelial cells by eliciting persistent Stat 3 signaling induced by autocrine leukemia inhibitory factor. Cancer Res. 2010; 70:8917-8926.
23. Xiong H, Du W, Zhang YJ, Hong J, Su WY, Tang JT, Wang YC, Lu R, Fang JY. Trichostatin A, a histone deacetylase inhibitor, suppresses JAK2/STAT3 signaling via inducing the promoter-associated histone acetylation of SOCS1 and SOCS3 in human colorectal cancer cells. Mol Carcinog. 2012; 51:174-184.

24. Fang J, Xu H, Yang C, Morsalin S, Kayarthodi S, Rungsrisuriyachai K, Gunnal U, Mckenzie B, Rao VN, Reddy SP. Ets Related Gene and Smad3 Proteins Collaborate to Activate Transforming Growth Factor-Beta Mediated Signaling Pathway in ETS Related Gene-Positive Prostate Cancer Cells. J Pharm Sci Pharmacol. 2014; 1:175-181.

25. Chen HS, Bai MH, Zhang T, Li GD, Liu M. Ellagic acid induces cell cycle arrest and apoptosis through TGF- $\beta$ / Smad3 signaling pathway in human breast cancer MCF-7 cells. Int J Oncol. 2015; 46:1730-1738.

26. Wang X, Liu C, Wang J, Fan Y, Wang Z, Wang Y. Oxymatrine inhibits the migration of human colorectal carcinoma RKO cells via inhibition of PAI-1 and the TGF- $\beta 1 /$ Smad signaling pathway. Oncol Rep. 2017; 37:747-753. 